\title{
Kalman Filter-Based Carrier Tracking with Ionospheric Scintillation Mitigation for GNSS
}

\author{
Rafael A. M. Lopes, Felix Antreich, and Hélio K. Kuga
}

\begin{abstract}
In this work we present two architectures of Kalman phase locked loop (PLL) to mitigate ionospheric scintillation induced effects on global navigation satellite systems (GNSS) receivers: a discriminator-based and an extended Kalman PLL using independent kinematic process models for the scintillation phase and amplitude to improve robustness in carrier synchronization. We show that the Kalman PLLs achieve advanced Doppler phase and scintillation amplitude estimation performance compared to an extended Kalman PLL with an autoregressive (AR) scintillation process model, with comparable scintillation phase estimation performance. The proposed Kalman PLLs have the additional benefits of not requiring parameter identification of $A R$ models.
\end{abstract}

Keywords-Ionospheric scintillation, GNSS, amplitude and phase estimation, Kalman PLL.

\section{INTRODUCTION}

Ionospheric scintillation is a phenomenon in which propagating electromagnetic waves interact with transient electron content inside regions of the ionosphere, introducing temporal fluctuations in the signal being transmitted via refraction and diffraction. For global navigation satellite systems (GNSS) in particular, ionospheric scintillation can have a significant impact on the availability, accuracy, continuity, and integrity of the positioning with such systems. The signal processing channels in a GNSS receiver perform carrier and code delay tracking of GNSS satellite signals. Regarding the received signal carrier, ionospheric scintillation is one source of amplitude and phase variations adding up to the line-of-sight (LOS) dynamics, introducing disturbances to GNSS tracking algorithms in the receiver that in many cases cause a reduction of precision in the positioning and eventually lead to the loss of lock of GNSS signals.

Especially in the carrier tracking, the estimation of the amplitude and phase variations induced by scintillation is important, on the one hand, to increase the tracking loop robustness to those effects, and on the other hand for scintillation detection and monitoring. Typically, the traditional tracking loop structures implemented with PLLs and/or frequency locked loops (FLL) are not directly providing estimates of scintillation amplitude or phase variations, thus not mitigating their effects in carrier synchronization. Indirect approaches to improve carrier synchronization robustness to scintillation include the adjustment of the tracking parameters such as noise

Rafael A. M. Lopes, Departamento de Mecânica e Controle, INPE, São José dos Campos-SP, e-mail: rafael.lopes@inpe.br; Felix Antreich, Departamento de Telecomunicações, ITA, São José dos Campos-SP, e-mail: antreich@ieee.org; Hélio K. Kuga, Departamento de Mecânica e Controle, INPE, São José dos Campos-SP, e-mail: helio.kuga@inpe.br. This work was partially funded by CNPq. bandwidth [1] in PLLs and the employment of a FLL-assisted PLL structure [2], for example.

The Kalman filter has been successfully employed in GNSS carrier tracking loops with this intent. The introduction of models representing the scintillation dynamics in the received signal into the Kalman filter formulation enabled decoupling both scintillation and LOS contributions to overcome the limitations of those indirect techniques that adjust the level of uncertainty in models accounting for states related to the LOS dynamics only. The LOS dynamics are typically modeled by a kinematic process [3] in the Kalman PLL and scintillation amplitude and phase are typically modeled by autoregressive (AR) processes [4], [5]. In this case, the parameters of the AR model also have to be estimated. In [6], an extended Kalman filter PLL structure with two outputs related to the inphase/quadrature (I/Q) branches and estimation of both scintillation amplitude and phase is employed, with the parameters of the AR model identified offline and adaptive measurement noise covariance update. This concept is generalized in [7] with an extended Kalman filter with increased complexity including online identification of the AR model parameters and online updating of the process noise covariance matrix based on the identification statistics.

In this work, we explore scintillation phase and amplitude estimation for scintillation mitigation. We present two Kalman filter PLLs for carrier synchronization with increased robustness to scintillation induced effects: a discriminatorbased Kalman PLL and an extended Kalman PLL. Both running in a receiver with a traditional noncoherent delay locked loop (DLL). The discriminator-based Kalman PLL model includes the dynamics of the scintillation phase and the extended Kalman PLL model includes the dynamics of scintillation phase and amplitude, in addition to the LOS dynamics in both cases. Scintillation phase and amplitude are modeled by a suitable kinematic process model, and are considered independent to the LOS dynamics, which are also modeled by a kinematic process model. Compared to the AR process model, this process model allows a simpler formulation, since parameter identification is not required. We show the capability of both structures by numerical simulations considering synthetic scintillation phase variations generated by the Cornell Scintillation Model (CSM) [8] added to a simulated baseband (BB) Global Positioning System (GPS) L1 C/A code signal and comparing the proposed algorithms' results regarding the estimation of scintillation induced effects and the LOS tracking to the results of state-of-the-art adaptive extended Kalman PLL with online parameter identification of the scintillation AR process model as defined in [7]. 


\section{Signal Model}

The received signal considered in this work is a GPS L1 C/A code signal of one GPS satellite. After downconversion to an intermediate frequency in the front-end and disregarding the noise, the GPS L1 C/A code signal from one satellite can be given as

$$
s(t)=A(t) g(t-\tau) c(t-\tau) \cos \left[2 \pi f_{I F} t+\phi_{T}(t)\right],
$$

where $A(t)$ is the amplitude, $g(t-\tau)$ is the navigation message with $g(t) \in\{-1,1\}, c(t-\tau)$ is the C/A code, $f_{I F}$ is the intermediate frequency (IF) in $\mathrm{Hz}, \tau$ is the time-delay, and $\phi_{T}(t)$ is the total phase, including Doppler phase, reference oscillator offset, and other sources of phase variations, such as ionospheric scintillation. This IF signal downconverted to baseband in the front-end prior to the analog-to-digital converter (ADC), considering unitary amplitude, no navigation bit transition, and a signal bandwidth $B$, has the following I/Q (Inphase/Quadrature) components

$$
\begin{aligned}
& I(t)=c(t-\tau) \cos \left[\phi_{T}(t)\right]=c(t-\tau) \cos \left[2 \pi f_{D} t+\phi(t)\right] \\
& Q(t)=c(t-\tau) \sin \left[\phi_{T}(t)\right]=c(t-\tau) \sin \left[2 \pi f_{D} t+\phi(t)\right],
\end{aligned}
$$

where $f_{D}$ is the Doppler frequency shift in $\mathrm{Hz}$ and $\phi(t)$ is the phase due to other sources of phase variations. Therefore, the total phase $\phi_{T}(t)$ of the carrier is composed of a term related to the Doppler shift and an independent term related to phase variations due to sources other than the LOS dynamics

$$
\phi_{T}(t)=\phi_{D}(t)+\phi(t) \text {. }
$$

The sampled complex input signal based on its I/Q components, considering unit amplitude, and no navigation bit transition, is given as

$$
s[n]=c[\tau[n]] e^{j \phi_{T[n]}}=c[n ; \tau[n]] e^{j \tan ^{-1}(Q[n] / I[n])},
$$

where $n=0, \ldots, N-1$ is the index relative to the ADC sampling such that $t=n T_{S}$ and $T_{S}=1 / f_{S}=1 / 2 B$, $c[n ; \tau[n]]$ is the discrete code sequence delayed by $\tau[n]$, and $I[n]$ and $Q[n]$ are the complex I/Q components, respectively.

Now, collecting $N$ samples of the signal in a vector

$$
\mathbf{s}[k]=[s[k N+0], s[k N+1], \ldots, s[k N+N-1]]^{\mathrm{T}}
$$

the digital input sequence (4) can be written as

$$
\mathbf{s}[k]=e^{j \phi[k]}\left(\mathbf{c}[\tau[k]] \odot \mathbf{d}\left[k, f_{D}[k]\right]\right) \in \mathbb{C}^{N \times 1},
$$

where $k=0, \ldots, K-1$ is the coherent integration index. The elements of $\mathbf{d}\left[k, f_{D}[k]\right]$ are $e^{j\left(f_{D}[k] k N T_{S}+\phi_{D}[k]\right)}$ for the Doppler frequency shift $f_{D}[k]$ in rad/s (observe the removal of the $2 \pi), \mathbf{c}[\tau[k]]$ is the vector formed by the GPS L1 code sequence $c[n ; \tau[n]]$ at $k$, and the binary operator $\odot$ represents element-by-element multiplication of two vectors (HadamardSchur product). In the same way, all internal tracking loop variables running at $f_{S}$ can also be represented as $N \times 1$ vectors.

Considering the complete discrete BB signal model $\mathbf{s}[k] \in$ $\mathbb{C}^{N \times 1}$, as given in (6) including the amplitude $A[k]$, the noise $\mathbf{n}[k]$, and disregarding the navigation bit $g[k]$, available in the receiver after the $\mathrm{ADC}$, we have

$$
\mathbf{s}[k]=A[k] e^{j \phi[k]}\left(\mathbf{c}[\tau[k]] \odot \mathbf{d}\left[k, f_{D}[k]\right]\right)+\mathbf{n}[k] .
$$

Discarding the noise term, the result of correlation after mixing with the carrier $\mathbf{d}\left[k, \hat{f}_{D}[k]\right]$ can be given by

$$
y[k]=\frac{1}{N} \mathbf{s}^{\mathrm{H}}[k]\left(\mathbf{c}[\hat{\tau}[k]] \odot \mathbf{d}\left[k, \hat{f}_{D}[k]\right]\right),
$$

where the superscript ${ }^{\mathrm{H}}$ represents the transposed complex conjugate. Developing the equation above, we get

$$
\begin{array}{r}
y[k]=\frac{A[k]}{N} e^{-j\left(\phi[k]+\phi_{D}[k]-\hat{\phi}_{D}[k]\right)}\left(\mathbf{c}^{\mathrm{H}}[\tau[k]] \odot \mathbf{c}[\hat{\tau}[k]]\right) \\
\left(\mathbf{d}^{\mathrm{H}}\left[k, f_{D}[k]\right] \odot \mathbf{d}\left[k, \hat{f}_{D}[k]\right]\right)+\eta_{D}[k] .
\end{array}
$$

The zero-mean complex white Gaussian noise $\eta_{D}[k]$ has variance $\sigma_{\eta_{D}}^{2}=\sigma_{n}^{2} / N$, where $\sigma_{n}^{2}=2 B N_{0}$ is the variance of $n[k N+n]$, the elements of the vector $\mathbf{n}[k]$. With $\delta \phi_{D}[k]=$ $\phi_{D}[k]-\hat{\phi}_{D}[k]$ and $\delta f_{D}[k]=f_{D}[k]-\hat{f}_{D}[k]$ we can derive an approximate expression for the prompt correlator output after mixing with the carrier generated by the receiver as ${ }^{1}$

$$
\begin{array}{r}
y[k]=A[k] \operatorname{sinc}\left(\frac{-\delta f_{D}[k] T_{s}}{2 \pi}\right) e^{-j\left(\phi[k]+\delta \phi_{D}[k]\right)} \\
e^{-j\left[(N-1) T_{s} / 2\right] \delta f_{D}[k]}+\eta_{D}[k] .
\end{array}
$$

If $\left|\delta f_{D}[k] T_{s}\right| \ll 1, \operatorname{sinc}\left(\frac{-\delta f_{D}[k] T_{S}}{2 \pi}\right) \approx 1$. For an unitary amplitude of the carrier undisturbed by scintillation, $A[k]$ is equal to the scintillation amplitude $\rho[k]$ and $(N-1) T_{s} \approx T_{I}$, so we can write the I/Q components of $y[k]=y_{I}[k]+j y_{Q}[k]$ as

$$
\left\{\begin{aligned}
y_{I}[k] & =\rho[k] \cos \left(-\phi[k]-\delta \phi_{D}[k]-\frac{T_{I}}{2} \delta f_{D}[k]\right) \\
& +\eta_{D_{I}}[k] \\
y_{Q}[k] & =\rho[k] \sin \left(-\phi[k]-\delta \phi_{D}[k]-\frac{T_{I}}{2} \delta f_{D}[k]\right) \\
& +\eta_{D_{Q}}[k]
\end{aligned}\right.
$$

with $\sigma_{\eta_{D_{I}}}^{2}=\sigma_{\eta_{D_{Q}}}^{2}=\sigma_{\eta_{D}}^{2} / 2$. Further simplifying by disregarding the term in $\delta f_{D}[k]$ leads to the nonlinear measurement equations commonly employed in the extended Kalman filter [6], [7] with

$$
\left\{\begin{aligned}
y_{I}[k] & =\rho[k] \cos \left(-\phi[k]-\delta \phi_{D}[k]\right)+\eta_{D_{I}}[k] \\
y_{Q}[k] & =\rho[k] \sin \left(-\phi[k]-\delta \phi_{D}[k]\right)+\eta_{D_{Q}}[k]
\end{aligned}\right.
$$

Application of the four quadrant arctangent $\varepsilon_{P}[k]=$ $-\operatorname{atan} 2\left[y_{Q}[k], y_{I}[k]\right]$ yields

$$
\varepsilon_{P}[k]=\phi[k]+\delta \phi_{D}[k]+\eta_{A}[k],
$$

which forms the innovations sequence for the discriminatorbased PLL, where $\eta_{A}[k]$ is the noise at the output of the four quadrant arctangent, with variance $\sigma_{\eta_{A}}^{2}$ that can be computed by approximate expression as shown in [9].

$$
\begin{aligned}
& \sum_{n=0}^{N-1} e^{j n z}=e^{j(N-1) z / 2} \frac{\sin (N z / 2)}{\sin (z / 2)} \approx N e^{j(N-1) z / 2} \operatorname{sinc}\left[\frac{z}{2 \pi}\right], \\
& \operatorname{sinc}(t)=\sin (\pi t) /(\pi t) \text { and } z=-\delta f_{D}[k] T_{s} .
\end{aligned}
$$




\section{KALMAN PLLs FOR Scintillation Mitigation}

The state vector to be estimated by the Kalman filter is $\mathbf{x}[k] \in \mathbb{R}^{P \times 1}$. In the more general case of the extended Kalman filter, the state evolution and the measurement equations are formed by nonlinear functions according to the discrete system equations

$$
\begin{aligned}
& \mathbf{x}[k]=\mathbf{f}(\mathbf{x}[k-1])+\boldsymbol{\nu}[k] \\
& \mathbf{y}[k]=\mathbf{h}(\mathbf{x}[k])+\mathbf{n}[k],
\end{aligned}
$$

where $\mathbf{f}(\cdot) \in \mathbb{R}^{P \times 1}$ and $\mathbf{h}(\cdot) \in \mathbb{R}^{M \times 1}$ are nonlinear functions of the state vector $\mathbf{x}[k] \in \mathbb{R}^{P \times 1}, \mathbf{y}[k] \in \mathbb{R}^{M \times 1}$ is the measurement vector, $\boldsymbol{\nu}[k] \in \mathbb{R}^{P \times 1}$ and $\mathbf{n}[k] \in \mathbb{R}^{M \times 1}$ are independent Gaussian process and measurement noises whose covariance matrices are $\mathbf{Q} \in \mathbb{R}^{P \times P}$ and $\mathbf{R} \in \mathbb{R}^{M \times M}$, respectively. The propagation step and the update step of the Kalman filter can be given as

$$
\begin{aligned}
\hat{\mathbf{x}}[k \mid k-1] & =\mathbf{f}(\hat{\mathbf{x}}[k-1]) \\
\mathbf{P}[k \mid k-1] & =\mathbf{F}[k-1] \mathbf{P}[k-1] \mathbf{F}^{\mathrm{T}}[k-1]+\mathbf{Q}
\end{aligned}
$$

and

$$
\begin{aligned}
\mathbf{K}[k] & =\mathbf{P}[k \mid k-1] \mathbf{H}^{\mathrm{T}}[k]\left(\mathbf{H}[k] \mathbf{P}[k \mid k-1] \mathbf{H}^{\mathrm{T}}[k]+\mathbf{R}\right)^{-1} \\
\mathbf{P}[k] & =(\mathbf{I}-\mathbf{K}[k] \mathbf{H}[k]) \mathbf{P}[k \mid k-1] \\
\hat{\mathbf{x}}[k] & =\hat{\mathbf{x}}[k \mid k-1]+\mathbf{K}[k]\{\mathbf{y}[k]-\mathbf{h}(\hat{\mathbf{x}}[k \mid k-1])\} .
\end{aligned}
$$

The measurement residual or innovation is $\varepsilon[k]=\mathbf{y}[k]-$ $\mathbf{h}(\hat{\mathbf{x}}[k \mid k-1]), \mathbf{P}[k] \in \mathbb{R}^{P \times P}$ is the state covariance matrix and $\mathbf{K}[k] \in \mathbb{R}^{P \times M}$ is the Kalman gain. In the case of the Kalman filter, the process and measurement equations are linear functions of the state vector, so that $\mathbf{f}(\mathbf{x}[k-1])=$ $\mathbf{F} \mathbf{x}[k-1]$ with $\mathbf{F} \in \mathbb{R}^{P \times P}$ and $\mathbf{h}(\mathbf{x}[k])=\mathbf{H} \mathbf{x}[k]$ with $\mathbf{H} \in \mathbb{R}^{M \times P}$, respectively. In the case of the extended Kalman filter, $\mathbf{f}(\mathbf{x}[k-1])$ and/or $\mathbf{h}(\mathbf{x}[k])$ are nonlinear functions of the states, whose Jacobians

$$
\mathbf{F}[k-1]=\left.\frac{\partial \mathbf{f}}{\partial \mathbf{x}}\right|_{\mathbf{x}=\hat{\mathbf{x}}[k-1]}, \mathbf{H}[k]=\left.\frac{\partial \mathbf{h}}{\partial \mathbf{x}}\right|_{\mathbf{x}=\hat{\mathbf{x}}[k \mid k-1]}
$$

are employed in the prediction and update equations of the extended Kalman filter.

Let the LOS dynamics and scintillation induced phase and amplitude be considered independent processes, and both LOS and scintillation are modeled by the kinematic process equations. Then the scintillation process model can be appended to the LOS process model, so that LOS robust tracking and scintillation phase and amplitude estimation are accomplished by a Kalman filter. Hence, we model the LOS, scintillation phase, and scintillation amplitude as third order Wiener processes. Therefore, for scintillation mitigation purposes we consider a Kalman PLL, whose LOS dynamics state-space equation can be given as

$$
\underbrace{\left[\begin{array}{c}
\phi_{D}[k] \\
f_{D}[k] \\
a_{D}[k]
\end{array}\right]}_{=\mathbf{x}_{D}[k]}=\underbrace{\left[\begin{array}{ccc}
1 & T_{I} & T_{I}^{2} / 2 \\
0 & 1 & T_{I} \\
0 & 0 & 1
\end{array}\right]}_{=\mathbf{F}_{D}} \underbrace{\left[\begin{array}{l}
\phi_{D}[k-1] \\
f_{D}[k-1] \\
a_{D}[k-1]
\end{array}\right]}_{=\mathbf{x}_{D}[k-1]}+\boldsymbol{\nu}_{D}[k],
$$

the scintillation phase process model is

$$
\underbrace{\left[\begin{array}{c}
\phi[k] \\
\dot{\phi}[k] \\
\ddot{\phi}[k]
\end{array}\right]}_{=\mathbf{x}_{\phi_{S}}[k]}=\underbrace{\left[\begin{array}{ccc}
1 & T_{I} & T_{I}^{2} / 2 \\
0 & 1 & T_{I} \\
0 & 0 & 1
\end{array}\right]}_{=\mathbf{F}_{\phi_{S}}} \underbrace{\left[\begin{array}{c}
\phi[k-1] \\
\dot{\phi}[k-1] \\
\ddot{\phi}[k-1]
\end{array}\right]}_{\mathbf{x}_{\phi_{S}}[k-1]}+\boldsymbol{\nu}_{\phi_{S}}[k],
$$

and the scintillation amplitude process model is

$$
\underbrace{\left[\begin{array}{c}
\rho[k] \\
\dot{\rho}[k] \\
\ddot{\rho}[k]
\end{array}\right]}_{=\mathbf{x}_{\rho_{S}}[k]}=\underbrace{\left[\begin{array}{ccc}
1 & T_{I} & T_{I}^{2} / 2 \\
0 & 1 & T_{I} \\
0 & 0 & 1
\end{array}\right]}_{=\mathbf{F}_{\rho_{S}}} \underbrace{\left[\begin{array}{c}
\rho[k-1] \\
\dot{\rho}[k-1] \\
\ddot{\rho}[k-1]
\end{array}\right]}_{\mathbf{x}_{\rho_{S}}[k-1]}+\boldsymbol{\nu}_{\rho_{S}}[k] .
$$

So the LOS state vector is formed by Doppler phase $\phi_{D}[k]$, frequency shift $f_{D}[k]$ and frequency drift $a_{D}[k]$. The scintillation phase state vector is formed by phase $\phi[k]$, phase velocity $\dot{\phi}[k]$ and phase acceleration $\ddot{\phi}[k]$ and the scintillation amplitude state vector is formed by amplitude $\rho[k]$, amplitude velocity $\dot{\rho}[k]$ and amplitude acceleration $\ddot{\rho}[k]$. The process noises $\boldsymbol{\nu}_{D}[k], \boldsymbol{\nu}_{\phi_{S}}[k]$, and $\boldsymbol{\nu}_{\rho_{S}}[k]$ are defined by the covariance matrices $\mathbf{Q}_{D}=\sigma_{a_{D}}^{2} \mathbf{Q}_{I}, \mathbf{Q}_{\phi_{S}}=\sigma_{\ddot{\phi}_{S}}^{2} \mathbf{Q}_{I}$ and $\mathbf{Q}_{\rho_{S}}=\sigma_{\ddot{\rho}_{S}}^{2} \mathbf{Q}_{I}$, respectively, where

$$
\mathbf{Q}_{I}=\left[\begin{array}{ccc}
T_{I}^{5} / 20 & T_{I}^{4} / 8 & T_{I}^{3} / 6 \\
T_{I}^{4} / 8 & T_{I}^{3} / 3 & T_{I}^{2} / 2 \\
T_{I}^{3} / 6 & T_{I}^{2} / 2 & T_{I}
\end{array}\right]
$$

The process noise covariance matrix $\mathbf{Q}_{D}$ for the LOS dynamics is typically defined by $\sigma_{a_{D}}^{2}=0.2 \mathrm{rad}^{2} / \mathrm{s}^{5}$ [5]. The variances $\sigma_{\ddot{\phi}_{S}}^{2}$ and $\sigma_{\ddot{\rho}_{S}}^{2}$ are experimentally adjusted based on the analysis of the data. We explore two formulations of the Kalman PLL employing the proposed kinematic models for the scintillation processes: the first one is discriminator-based, where the innovations are computed directly by the phase discriminator. In this case, the scintillation amplitude is not part of the state vector now given as

$$
\mathbf{x}_{1}[k]=\left[\begin{array}{ll}
\mathbf{x}_{D}^{\mathrm{T}}[k] & \mathbf{x}_{\phi_{S}}^{\mathrm{T}}[k]
\end{array}\right]^{\mathrm{T}} .
$$

The second one is an extended Kalman filter, with the complete state vector

$$
\mathbf{x}_{2}[k]=\left[\begin{array}{lll}
\mathbf{x}_{D}^{\mathrm{T}}[k] & \mathbf{x}_{\phi_{S}}^{\mathrm{T}}[k] & \mathbf{x}_{\rho_{S}}^{\mathrm{T}}[k]
\end{array}\right]^{\mathrm{T}} .
$$

The state transition matrices $\mathbf{F}_{1}$ and $\mathbf{F}_{2}$ for, respectively, $\mathbf{x}_{1}$ and $\mathbf{x}_{2}$, are

$$
\mathbf{F}_{1}=\left[\begin{array}{cc}
\mathbf{F}_{D} & \mathbf{0} \\
\mathbf{0} & \mathbf{F}_{\phi_{S}}
\end{array}\right], \quad \mathbf{F}_{2}=\left[\begin{array}{ccc}
\mathbf{F}_{D} & \mathbf{0} & \mathbf{0} \\
\mathbf{0} & \mathbf{F}_{\phi_{S}} & \mathbf{0} \\
\mathbf{0} & \mathbf{0} & \mathbf{F}_{\rho_{S}}
\end{array}\right]
$$

with the respective covariance matrices

$$
\mathbf{Q}_{1}=\left[\begin{array}{cc}
\mathbf{Q}_{D} & \mathbf{0} \\
\mathbf{0} & \mathbf{Q}_{\phi_{S}}
\end{array}\right], \quad \mathbf{Q}_{2}=\left[\begin{array}{ccc}
\mathbf{Q}_{D} & \mathbf{0} & \mathbf{0} \\
\mathbf{0} & \mathbf{Q}_{\phi_{S}} & \mathbf{0} \\
\mathbf{0} & \mathbf{0} & \mathbf{Q}_{\rho_{S}}
\end{array}\right]
$$

In the discriminator-based Kalman filter, the innovations are directly computed by (13), so that

$$
\mathbf{H}_{1}=\left[\begin{array}{llllll}
1 & 0 & 0 & 1 & 0 & 0
\end{array}\right] .
$$


In case of the extended Kalman filter, we have (12) as the nonlinear observation equations, whose linearization leads to

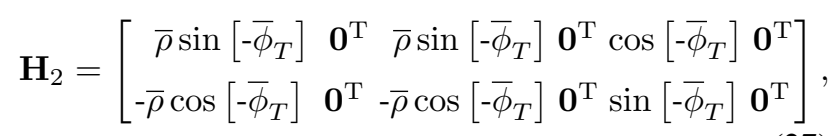

where $\bar{\rho}=\rho[k \mid k-1]$ and $\bar{\phi}_{T}=\phi[k \mid k-1]+\phi_{D}[k \mid k-$ $1]$, from the prediction step of the Kalman filter, and $\mathbf{0}=$ $\left[\begin{array}{ll}0 & 0\end{array}\right]^{\mathrm{T}}$. The measurement noise covariance matrix for the discriminator-based filter is defined by the atan 2 discriminator noise approximation [9]

$$
\mathbf{R}_{1}=\frac{1}{2 c / n_{0} T_{I}}\left(1+\frac{1}{c / n_{0} T_{I}}\right),
$$

where $c / n_{0}$ is the carrier to noise ratio in $\mathrm{Hz}$, obtained from $C / N_{0}$ in $\mathrm{dB}-\mathrm{Hz}$ as $c / n_{0}=10^{C / N_{0} / 10}$. We define $\mathbf{R}_{1}$ by fixing the value of the carrier to noise ratio at the relatively low value of $C / N_{0}=25 \mathrm{~dB}-\mathrm{Hz}$, so the covariance is adjusted for weaker signals but is high enough to cover stronger signals above this level. The discriminator-based filter is chosen to be non-adaptive so we can have a simpler model, to be compared to the more complex adaptive filters. The measurement noise covariance matrix $\mathbf{R}_{2}$ for the extended Kalman filter is adaptively adjusted based on $C / N_{0}$ estimation, according to [6].

\section{Simulations}

The discriminator-based and extended Kalman PLLs proposed for scintillation mitigation are evaluated by numerical simulations considering synthetic scintillation input data generated by the CSM [8] in a severe scintillation scenario defined by amplitude strength index $\mathrm{S}_{4}=0.8$ and decorrelation time $\tau_{0}=0.1$. The $\mathrm{S}_{4}$-index is the variational coefficient of the signal amplitude or power [10], and $\tau_{0}$ is the scintillation decorrelation time, a measure of the rapidness of the scintillation. A small $\tau_{0}$ (e.g., $<0.5 \mathrm{~s}$ ) implies a scintillating channel that changes rapidly with time [11]. We consider a maximum Doppler frequency drift of $0.94 \mathrm{~Hz} / \mathrm{s}$ that an on-ground static receiver would experience based on the GPS satellites' orbital dynamics, added to the CSM scintillation variation data.

The receiver parameters common to all simulations are shown in Table I. The receiver running at $f_{S}=2 B=4.092$ $\mathrm{MHz}$ and with coherent integration time of $T_{I}=1 \mathrm{~ms}$ integrates $N=f_{S} T_{I}=4092$ signal samples per cycle. No navigation bit transitions are considered and the undisturbed carrier amplitude is unitary. The same realization of a Gaussian noise sequence is added to the sampled I/Q signal components in all simulations, resulting in the noise power $2 B N_{0}$ for $C / N_{0}=45 \mathrm{~dB}-\mathrm{Hz}$. We consider receiving only one satellite signal with the pseudorandom noise sequence PRN $=1$. The results are presented in terms of the root mean square error (RMSE)

$$
\operatorname{RMSE}_{x}[k]=\sqrt{\frac{1}{k} \sum_{i=1}^{k}(x[k]-\hat{x}[k])^{2}},
$$

with $x$ being substituted by the analyzed parameters. In
TABLE I

RECEIVER TRACKING LOOP PARAMETERS. GPS C/A CODE SIGNAL.

\begin{tabular}{|c|c|}
\hline Sampling frequency $\left(f_{S}\right)$ & $4.092 \mathrm{MHz}$ \\
\hline Pseudorandom noise (PRN) sequence & 1 \\
\hline Early-late correlator spacing $(\Delta \mathrm{Tc})$ & $0.5 \mathrm{chip}$ \\
\hline Coherent integration time & $1 \mathrm{~ms}$ \\
\hline
\end{tabular}

the following we label the mitigation algorithm based on the discriminator-based PLL and kinematic process models as KPLL-sKIN, and the mitigation algorithm based on the extended Kalman PLL with kinematic process models and adaptive measurement covariance matrix as EKPLL-sKIN$A D A P T$. As a benchmark algorithm we consider the adaptive extended Kalman PLL with AR scintillation model presented in [7] and we label this algorithm as EKPLL-sAR-ADAPT. In all cases the DLL's second order loop filter is set with damping ratio $\xi=1 / \sqrt{2}$ and noise bandwidth $B_{n_{D L L}}=0.02$ $\mathrm{Hz}$, employing extended correlators [12] such that early and late correlators use 20 noncoherent correlations.

The results of the proposed algorithms are compared to the results of the EKPLL-SAR-ADAPT with online parameter identification via recursive least squares [13] with 2000 points sliding window [14] following the approach presented in [7]. The RMSE of the scintillation phase $\phi[k]$, the scintillation amplitude $\rho[k]$, and the Doppler phase $\phi_{D}[k]$ for the different algorithms are presented in Figures 1, 2 and 3, respectively.

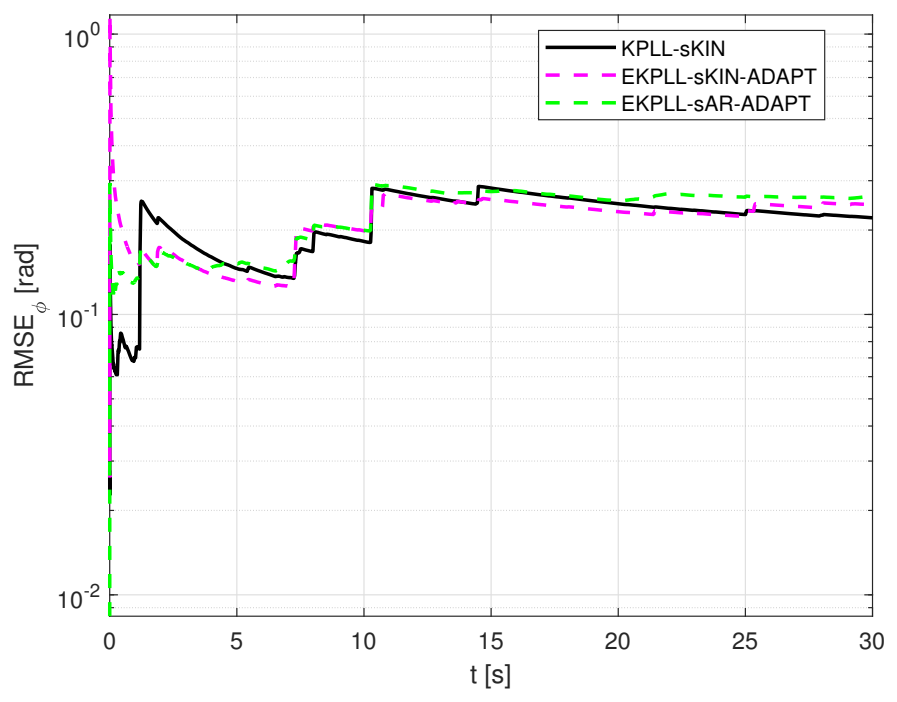

Fig. 1. Scintillation phase estimation $\mathrm{RMSE}_{\phi}$.

Regarding scintillation estimation, in Figure 1 we can observe that the $\mathrm{RMSE}_{\phi}$ for all algorithms is comparable. In Figure 2 we can see that as the extended Kalman filter formulations of EKPLL-sKIN-ADAPT and EKPLL-sAR-ADAPT include the scintillation amplitude in their processes models, they present a smaller $\mathrm{RMSE}_{\rho}$ compared to KPLL-sKIN, with the $\mathrm{RMSE}_{\rho}$ of the EKPLL-sKIN-ADAPT being smaller than the of the benchmark algorithm EKPLL-SAR-ADAPT. The amplitude estimate for the discriminator-based algorithm is computed as $\hat{\rho}[k]=\sqrt{I_{P}^{2}[k]+Q_{P}^{2}[k]}$. The Kalman PLLs perform better with respect to scintillation mitigation, as shown in Figure 3, with both the KPLL-sKIN and the EKPLL- 


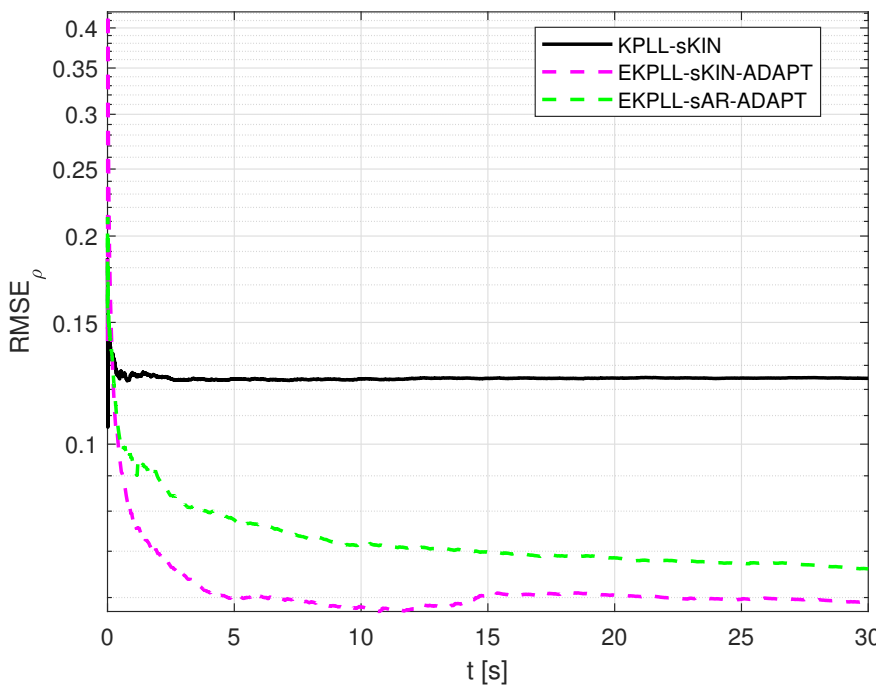

Fig. 2. Scintillation amplitude estimation $\operatorname{RMSE}_{\rho}$.

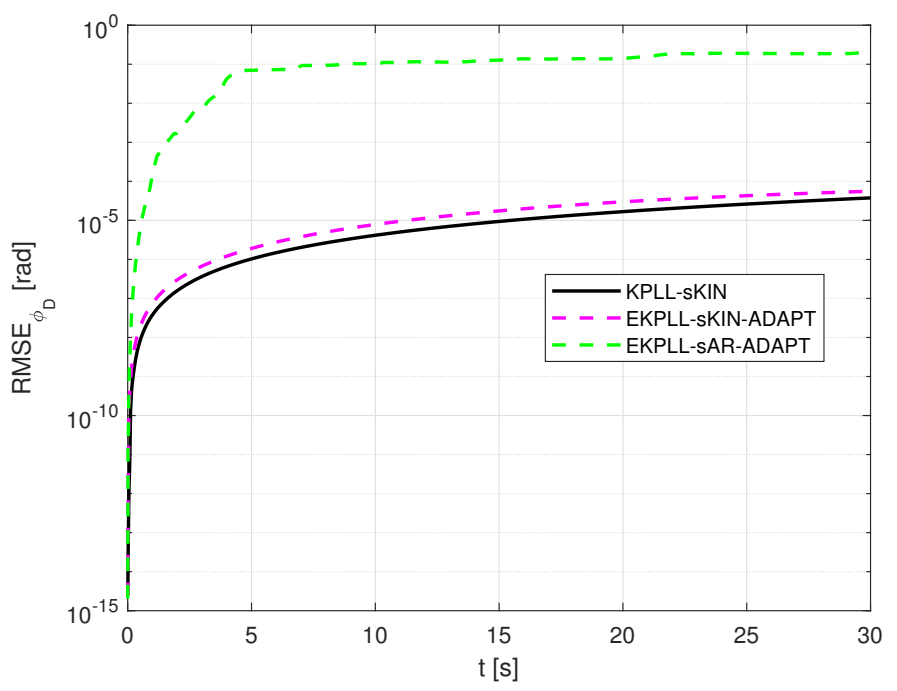

Fig. 3. Doppler phase estimation $\mathrm{RMSE}_{\phi_{D}}$.

$s K I N-A D A P T$ presenting smaller $\mathrm{RMSE}_{\phi_{D}}$ when compared to the benchmark extended Kalman filter with online parameters identification called EKPLL-SAR-ADAPT.

\section{CONCLUSion}

We presented a discriminator-based and an adaptive extended Kalman PLL, both with scintillation modeled using a suitable kinematic process model. The algorithms were evaluated via simulations with synthetic ionospheric scintillation data. The proposed adaptive extended Kalman PLL presented smaller errors when compared to the considered benchmark adaptive extended Kalman PLL with online identification of the AR scintillation model [7]. Both proposed Kalman PLLs presented smaller error in LOS tracking than the benchmark approach given in [7], showing good mitigation characteristics of the scintillation effects on the LOS dynamics tracking. Adding to this, the structures of the proposed approaches are simpler than of the benchmark algorithm, since parameter identification of the scintillation process model is not required.

\section{ACKNOWLEDGMENT}

This work was partially supported by the Brazilian National Council for Scientific and Technological Development (CNPq) under grant 309248/2018-3 PQ-2, 409865/2018-4, 307255/2018-2 and 205906/2018-4.

\section{REFERENCES}

[1] F. Legrand, C. Macabiau, J.-L. Issler, L. Lestarquit, and C. Mehlen, "Improvement of pseudorange measurements accuracy by using fast adaptive bandwidth lock loops," ION GPS 2000, pp. 2346 - 2356, Salt Lake City, United States, 2000.

[2] R. Xu, Z. Liu, and W. Chen, "Improved FLL-assisted PLL with in-phase pre-filtering to mitigate amplitude scintillation effects," GPS Solutions, v. 19, pp. 263-276, 2015.

[3] Bar-Shalom, Y., Li, X. R., Kirubarajan, T., Estimation with Applications to Tracking and Navigation, John Wiley and Sons, USA, 2001.

[4] J. Vilá-Valls, C. Fernandez-Prades, J. López-Salcedo, and G. SecoGranados, "Adaptive GNSS Carrier Tracking Under Ionospheric Scintillation: Estimation vs. Mitigation," IEEE Communications Letters, v. 19, pp. 961-964, 2015.

[5] F. Fohlmeister, F. Antreich and J. A. Nossek, "Dual Kalman filtering based GNSS phase tracking for scintillation mitigation," IEEE/ION Position, Location and Navigation Symposium (PLANS), Monterey, CA, 2018, pp. 1151-1158, 2018.

[6] J. Vilá-Valls, P. Closas, C. Fernandez-Prades and J.T. Curran, "On the Ionospheric Scintillation Mitigation in Advanced GNSS Receivers," IEEE Transactions on Aerospace and Electronic Systems, v. 54, no. 4, pp. 1692-1708, 2018.

[7] J. Vilá-Valls, C. Fernandez-Prades, J. Arribas, J.T. Curran and P. Closas, "On-line Model Learning for Adaptive GNSS Ionospheric Scintillation Estimation and Mitigation," IEEE/ION Position, Location and Navigation Symposium (PLANS), Monterey, CA, 2018, pp. 1167-1172, 2018.

[8] T.E. Humphreys, M.L. Psiaki, P.M. Kintner, "Modeling the effects of ionospheric scintillation on GPS carrier phase tracking", IEEE Trans. Aerosp. Electron. Syst., v. 46(4), pp. 1624-1637, 2010.

[9] Z. Luo, J. Ding, L. Zhao and M. Wu, "An Enhanced Non-Coherent Pre-Filter Design for Tracking Error Estimation in GNSS Receivers," Sensors (Basel), v. 17, no. 11, pp. 1-23, 2017.

[10] C. Hegarty, M.B. El-Arini, T. Kim, S. Ericson, "Scintillation modeling for GPS-wide area augmentation system receivers", Radio Sci., v. 36(2), pp. 1221-1231, 2011.

[11] Teunissen, P. J. G. and Montenbruck, O., Springer Handbook of Global Navigation Satellite Systems, Spring International, Switzerland, 2017.

[12] M. Susi, D. Borio, "Kalman Filtering with Noncoherent Integrations for Galileo E6-B Tracking," Navigation, v. 67, issue 3, pp. 601-618, 2020

[13] T. Soderstrom and P. Stoica: System Identification, Prentice-Hall International, Hemel Hempstead, UK, 1989.

[14] P. C. Young, Recursive Estimation and Time-Series Analysis, second edition, Springer-Verlag, Germany, 2011. 